Check for updates

Cite this: RSC Adv., 2019, 9, 24733

Received 7th May 2019

Accepted 25th July 2019

DOI: $10.1039 / \mathrm{c} 9 \mathrm{ra03408k}$

rsc.li/rsc-advances

\section{Optimizing electron-rich arylamine derivatives in thiophene-fused derivatives as $\pi$ bridge-based hole transporting materials for perovskite solar cells $\uparrow$}

\begin{abstract}
Xiaorui Liu (ID* and Xing Liu (DD
Based on the observations of thienothiophene derivatives as $\pi$-bridged small molecule hole transporting materials (HTMs), adjusting their electron-rich arylamine derivatives is an effective approach to obtain the alternative HTMs for perovskite solar cells (PSCs). In this work, starting from a new electron-rich arylamine derivative and different $\pi$-bridged units of thienothiophene derivatives, a series of arylamine derivative-based HTMs were designed, and their properties were investigated using density functional theory combined with the Marcus charge transfer theory. Compared with the parental Z26 material, the designed $\mathrm{H01-H04}$ exhibit appropriate frontier molecular orbitals, good optical properties, better solubility, good stability and higher hole mobilities. $\mathrm{H01}-\mathrm{H0} 4$ materials with high hole mobility $\left(\sim \times 10^{-2}\right)$ can serve as promising HTMs for improving the efficiency of PSCs. The results confirm that the design strategy of adjusting the electron-rich arylamine derivatives in thienothiophene derivatives as $\pi$-bridged HTMs is a reliable approach to obtain the promising HTMs for PSC applications.
\end{abstract}

\section{Introduction}

Organic-inorganic hybrid perovskite materials (e.g., $\mathrm{CH}_{3} \mathrm{NH}_{3}-$ $\mathrm{PbX}_{3}, \mathrm{X}=\mathrm{Cl}, \mathrm{Br}$ and I) as light absorbers have excellent optoelectronic properties such as broad optical absorption, tunable band gaps, long charge-diffusion length and ambipolar charge transporting behaviour. ${ }^{1-3} \mathrm{Up}$ to now, the power conversion efficiency (PCE) of perovskite solar cells (PSCs) on the basis of a fluorene-terminated HTM has exceeded 23\%. ${ }^{4}$ In general, the PSC device architecture of indium tin oxide (ITO)/electrontransporting layer (ETL)/perovskite $\left(\mathrm{CH}_{3} \mathrm{NH}_{3} \mathrm{PbI}_{3}\right) /$ holetransporting layer (HTL)/Au is the most commonly used. ${ }^{5-7}$ As has been demonstrated, HTMs as the HTLs play a key role in hole extraction and transportation to $\mathrm{Au}$ electrodes for conventional devices. ${ }^{5}$ It is reported that the organic HTMs have been considered one of the most important factors restricting the device stability. ${ }^{8,9}$ In general, spiro-OMeTAD is used as a standard HTM to be widely used in traditional PSCs. Owing to the poor conductivity $\left(9.1 \times 10^{-7} \mathrm{~S} \mathrm{~cm}^{-1}\right)$ of spiro-OMeTAD itself, lithium bis-trifluoromethanesulfonyl imide (LiTFSI) and tert-butylpyridine (TBP) must be added to improve the conductivity. ${ }^{9,10}$ However, the dopants LiTFSI and TBP in HTL are the main factors that lead to the degradation of perovskites as LiTFSI tends to absorb water., ${ }^{\mathbf{9 1 0}}$ To overcome the

Key Laboratory of Luminescent and Real-Time Analytical Chemistry (Southwest University), Ministry of Education, School of Chemistry and Chemical Engineering, Southwest University, Chongqing 400715, China.E-mail: liuxiaorui@swu.edu.cn

$\dagger$ Electronic supplementary information (ESI) available: Table S1-S4, Fig. S1-S6. See DOI: $10.1039 / \mathrm{c} 9 \mathrm{ra03408 \textrm {k }}$ shortcomings of doped spiro-OMeTAD, the development of alternative HTMs with high hole transporting ability and good stability is important.

At present, diphenylamine derivatives [e.g., bis(4methoxyphenyl)amine] and triphenylamine derivative [e.g., 4methoxy- $N$-(4-methoxyphenyl)- $N$-phenylbenzenamine] are widely used as electron-rich units in the construction of HTMs, such as FDT, ${ }^{11} \mathrm{Z}_{26},{ }^{12} \mathrm{H} 101,{ }^{13} \mathrm{X} 59,{ }^{14}$ TQ2 (ref. 15) and TTH101..$^{5,16-18}$ In the aforementioned HTMs, adjusting the electron-deficient $\pi$-bridged units of diphenylamine or triphenylamine derivative-based HTMs is the usual method to improve their performance. For example, Sun and coworkers ${ }^{19,20}$ reported a class of star HTMs that were theoretically designed via adjusting the electron-deficient cores in order to obtain efficient HTMs with appropriate energy levels and high hole mobility for PSC applications. In fact, electron rich arylamine derivatives are also important parts that affect the properties of HTMs. For example, Jang and co-workers ${ }^{21}$ reported two HTMs (pDPA-DBTP and pTPA-DBTP), which contained the electron-deficient $\operatorname{di}\left(1\right.$-benzothieno) $\left[3,2-b: 2^{\prime}, 3^{\prime}-d\right]$ pyrrole (DBTP) as the $\pi$-bridged units and electron-rich units of the diphenylamine derivative and triphenylamine derivative. The PCEs of PSCs based on HTMs of pDPA-DBTP and pTPADBTP are $14.10 \%$ and $15.63 \%$, respectively. ${ }^{21}$ Moreover, based on the investigations via density-functional theory (DFT) and Marcus theory, Li and co-workers ${ }^{22-24}$ reported that the introduction of a highly symmetrical core to diphenylamine- or triphenylamine-derivative-based HTMs resulted in some organic HTMs with suitable energy levels and improved hole mobility. Therefore, modifying the electron rich arylamine 
derivatives of HTMs may be a reliable approach to obtain promising HTMs in PSC applications.

Herein, on the basis of the potential compounds such as thiophene, thienothiophene, dithienothiophene and terthienothiophene as electron-deficient $\pi$-bridged units, a strategy was devised to introduce the new electron-rich arylamine $[N, N$ bis(4-methoxyphenyl)naphthalen-2-amine] derivatives to construct HTMs (see H01-H04 in Fig. 1) in order to provide theoretical models and obtain the alternative HTMs. These electron-deficient $\pi$-bridged units with good photoelectronic properties have been proved in previous works. ${ }^{24-26}$ Moreover, Z26 materials in Fig. S1† reported by Grätzel and co-workers were chosen as a reference. ${ }^{\mathbf{1 2}}$ Z26 as a HTM in PSC applications exhibits good performance such as a hole mobility of 1.34 $\times 10^{-4} \mathrm{~cm}^{2} \mathrm{~V}^{-1} \mathrm{~s}^{-1}$ and PCE of $20.1 \%{ }^{12}$ Thereupon, the properties of these designed HTMs, such as their geometric structures, frontier molecular orbitals and reorganization energies were investigated by DFT. The optical properties of absorption and emission were calculated using time-dependent DFT (TDDFT). The electron coupling and hole mobility were predicted on the basis of the first-principle calculations combined with Marcus theory.

\section{Computational details}

\subsection{Geometry optimization and electronic properties}

Investigated systems are shown in Fig. 1 and $\mathrm{S} 1 . \dagger$ Geometric structures and electronic properties of the investigated systems were fully optimized using B3P86 at 6-311G(d,p) and BMK/6$31 \mathrm{G}(\mathrm{d})$ in a gaseous phase or tetrahydrofuran solution. ${ }^{27}$ The energies of all of the obtained geometries are ensured to be the lowest as the optimized structures do not exhibit imaginary frequency. In fact, frontier orbital energies of an organic molecule are very sensitive to the functionals and basis sets. For the arylamine derivative-based HTMs (Z26), HOMO energy was calculated using the methods of B3LYP, B3P86 and PBE0 at 6$311 \mathrm{G}(\mathrm{d}, \mathrm{p})$ levels. The calculated results indicate that the calculated HOMO energy (-5.05 eV) of Z26 with B3P86/6$311 \mathrm{G}(\mathrm{d}, \mathrm{p})$ functional and basis set is close to its experimental result ( $-5.16 \mathrm{eV}$ (ref. 12)). The HOMO energies of $\mathrm{Z} 26$ from the B3LYP/6-311G(d,p) and PBE0/6-311G(d,p) calculations are

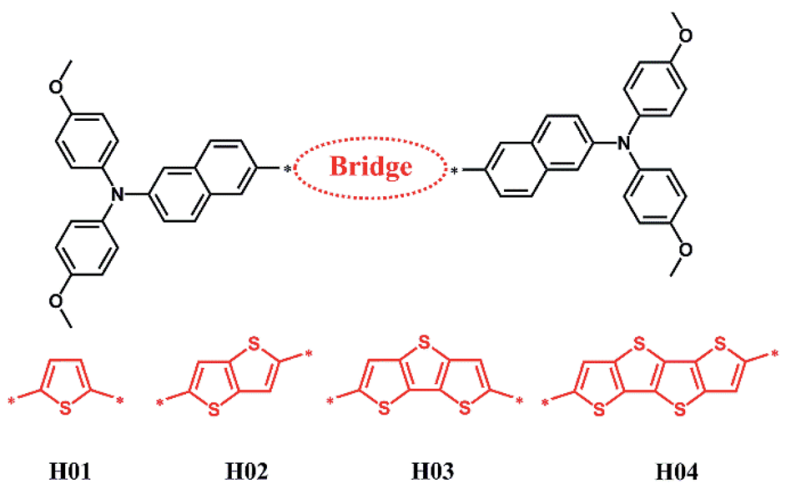

Fig. 1 Chemical structures of designed molecules $\mathrm{H} 01-\mathrm{H} 04$.
-4.45 and $-4.61 \mathrm{eV}$, respectively. Moreover, the calculated HOMO energy $(-5.09 \mathrm{eV})$ of FDT with B3P86/6-311G(d,p) functional and basis set is in line with its experimental result $(-5.16 \mathrm{eV}$ (ref. 28)). Therefore, the frontier orbital energies of the designed HTMs are calculated using DFT with B3P86/6$311 \mathrm{G}(\mathrm{d}, \mathrm{p})$ functional and basis set.

\subsection{Calculation of optical absorption}

On the basis of the ground-state geometries from the B3P86, BMK, B3LYP and PBE0 at 6-31G(d) level in tetrahydrofuran solution, the optical absorptions of $\mathrm{Z} 26$ were simulated by TDDFT with B3P86, BMK, B3LYP and PBE0 at 6-31G(d) level in tetrahydrofuran solution with a polarizable continuum model (PCM). ${ }^{27,29}$ Compared with the experimental value (456 nm (ref. 12)) of Z26, using the TD-BMK/6-31G(d) method in tetrahydrofuran solution could yield an accurate absorption peak at $450 \mathrm{~nm}$. Under the same conditions, the maximum absorption of Z26 obtained using the TD-B3LYP (529 nm), TD-PBE0 (530 $\mathrm{nm})$ and TD-B3P86 (507 $\mathrm{nm})$ methods are overestimated. Therefore, the optical absorptions of designed HTMs are calculated by TD-BMK/6-31G(d) on the basis of the same functional and basis set in tetrahydrofuran solution. The calculation of electron density difference including the overlap between the regions of density depletion and the increment and transferred charge amount can be obtained from our previous work. ${ }^{30}$ The DFT and TD-DFT calculations were carried out by the Gaussian 09 program. $^{31}$

\subsection{Energy calculations}

On the basis of the adiabatic potential energy surfaces, the inner reorganization energy $\lambda_{h}$ for holes of HTMs could be calculated as follows: $:^{25,32,33}$

$$
\lambda_{\mathrm{h}}=\left(E^{*}-E\right)+\left(E_{+}^{*}-E_{+}\right)
$$

where $E$ and $E_{+}$represent the energies of the neutral and cationic molecules in their lowest-energy geometries, respectively. $E^{*}$ and $E_{+}^{*}$ are the energies of the neutral and cationic states with the geometries of the cationic and neutral species. ${ }^{34}$ Moreover, energy calculations, including electron affinities, adiabatic ionization potential and absolute hardness of the investigated systems, were performed using the B3P86/6$311 G(d, p)$ method. The solvation free energy for all the molecules were calculated using the TD-BMK/6-31G(d) method in tetrahydrofuran solution or in a gaseous phase. Geometry optimization and single point energy calculations were implemented using the Gaussian 09 program.

\subsection{Calculations of charge mobility}

The hole mobility of the designed HTMs was calculated from the Einstein relation: ${ }^{35,36}$

$$
u_{\mathrm{h}}=\frac{e W}{k_{\mathrm{B}} T}
$$

where $e, W, k_{\mathrm{B}}$ and $T$ are the electron charge, charge diffusion coefficient, Boltzmann constant and temperature in Kelvin, respectively. For an $n$-dimensional system, $W$ is defined as the 
ratio between the mean-square displacement and the diffusion time: ${ }^{37}$

$$
W=\lim _{t \rightarrow \infty} \frac{1}{2 n} \frac{\left\langle x(t)^{2}\right\rangle}{t}
$$

For a spatially isotropic system, the homogeneous diffusion constant $W$ can be approximately evaluated by: ${ }^{38}$

$$
W=\frac{1}{2 n} \sum_{i} r_{i}^{2} k_{i} p_{i}
$$

where $i$ runs over all nearest adjacent molecules. The parameters such as $n, r_{i}, k_{i}$ and $p_{i}$ are the spatial dimensionality, the hopping distance, charge transfer rate $(k)$ and hopping probability $\left(p_{i}=k_{i} / \sum_{i} k_{i}\right)$, respectively.

Here, the parameter of hole transfer rate $\left(k_{\mathrm{h}}\right)$ for organic molecules can be calculated from the Marcus-Hush equation: ${ }^{36,39,40}$

$$
k_{\mathrm{h}}=\frac{\nu_{\mathrm{h}}^{2}}{h} \sqrt{\frac{4 \pi^{3}}{\lambda_{\mathrm{h}} k_{\mathrm{B}} T}} \exp \left(\frac{-\lambda_{\mathrm{h}}}{4 k_{\mathrm{B}} T}\right)
$$

where $v_{\mathrm{h}}, h$ and $\lambda_{\mathrm{h}}$ are the electronic coupling, Planck's constant and reorganization energy, respectively. Descriptions of charge transfer on the basis of the hopping mechanism is universally accepted. ${ }^{41-43}$ In eqn (5), the parameters such as $\lambda_{\mathrm{h}}$ and $v_{\mathrm{h}}$ are the key factors to determine the transfer rate of organic materials. The parameter of $\lambda_{\mathrm{h}}$ could be calculated from eqn (1).

The parameter of hole electronic coupling $\left(v_{\mathrm{h}}\right)$ could be obtained from the equation as shown below: ${ }^{44,45}$

$$
\nu_{\mathrm{h}}=\frac{J-S\left(H_{1}+H_{2}\right) / 2}{1-S^{2}}
$$

where $S, J$ and $H$ are the spatial overlap, charge transfer integral and site energies. The parameter of $J$ could be simulated by using the equation as shown below: ${ }^{44,45}$

$$
J=\left\langle\varphi_{\text {HOMO }}^{1}\left|h_{\mathrm{ks}}\right| \varphi_{\text {HOMO }}^{2}\right\rangle
$$

where $h_{\mathrm{ks}}$ is the Kohn-Sham Hamiltonian between two fragments. The parameters such as $\varphi_{\text {Номо }}^{1}$ and $\varphi_{\text {Номо }}^{2}$ are the HOMOs of the two fragments. The electronic coupling could be simulated from the PW91/TZP levels in the ADF program. ${ }^{46-49}$

\subsection{Simulation of crystal structures}

In order to calculate the electronic coupling of HTMs, it was necessary to obtain the dimer structure, which was defined as adjacent segments from the crystal structures of molecules. The crystal structure of the investigated HTMs can be obtained from the polymorph predictor module in the Material Studio software. ${ }^{50,51}$ The geometry of the cluster models used in the present study was taken from the B3P86/6-311G(d,p) level. The Dreiding force field was used for the prediction. ${ }^{52}$ For the investigated HTMs, the polymorph calculations are restricted to the ten most probable space groups, namely, $C_{2}, P 2_{1} / c, P 1, P 2_{1} 2_{1} 2_{1}, C 2 / c, P 2_{1}$, $P b c a, C c, P n a 2_{1}$ and $P b c n .{ }^{53}$ To verify the rationality of the selected models herein, the FDT crystal structure was predicted using the same methods. The simulated results indicate that the predicted FDT crystal structure share the space group of $P_{1}$, consistent with the experimental one. ${ }^{28}$ This validates that the calculation method is reasonable to a certain degree. Further, the crystal structures were sorted according to their total energy. On the basis of the crystal structures, we selected a molecule as the center. All of the adjacent fragments with the center are defined as the transport pathways. Thus, each transport pathway is the paired dimer between neighboring and center molecules.

\section{Results and discussion}

\subsection{Molecular design and electronic properties}

In PSC devices, the conventional architecture of indium tin oxide(ITO)/electron-transporting layer (ETL)/perovskite/holetransporting layer (HTL)/Au is the most commonly used. ${ }^{6,7}$ As has been demonstrated, HTMs as the HTL play a key role in hole extraction and transportation to the Au electrode in conventional devices. ${ }^{5}$ However, the frontier molecular orbitals are the basic parameters to estimate the properties of the HTMs in PSCs. For example, the HTMs should have an energy higher than that of the valence band for $\mathrm{CH}_{3} \mathrm{NH}_{3} \mathrm{PbI}_{3}(-5.43 \mathrm{eV}$ (ref. 54 and 55)) to ensure the hole injection or transfer on the perovskite/HTM interface. Analogously, to prevent the electron movement from the absorber (e.g., $\mathrm{CH}_{3} \mathrm{NH}_{3} \mathrm{PbBr}_{3}$ ) to the metal electrodes, the HTMs should have a higher lowest unoccupied molecular orbital (LUMO) level than the conduction band of $\mathrm{CH}_{3} \mathrm{NH}_{3} \mathrm{PbI}_{3}$ (-3.93 eV (ref. 54)). To further improve the HTM performances in PSC devices, the first aim of this work is to introduce the new electron-rich arylamine derivatives to construct HTMs with a suitable frontier molecular orbital and high hole mobility. Therefore, starting from the new electronrich arylamine derivatives and different $\pi$-bridged units of thienothiophene derivatives, a series of arylamine derivativebased HTMs were designed (as shown in Fig. 1) on the basis of the structure of the reported Z26 material (as shown in Fig. S1†). Optimized ground-state geometries of HTMs are presented in Fig. S2.†

The functional and basis set of B3P86/6-311G(d,p) can reproduce accurate HOMO energies for the reported Z26 and FDT. To further ensure the accurate HOMO and LUMO energies with which we can evaluate the electronic properties of the designed HTMs in PSC devices, we calculated the values of HOMO and LUMO energies by employing the B3P86/6$311 \mathrm{G}(\mathrm{d}, \mathrm{p})$ functional and basis set for 13 arylamine derivativebased HTMs. The detailed information on the HTMs in PSC applications that have been reported are listed in Table S1. $\dagger$ Based on the calculated results, important information regarding the theoretical and experimental HOMO and LUMO values for these HTMs were obtained. The data are presented in Table S1. $\dagger$ A correlation can be established between the theoretical HOMO/LUMO energy levels and the experimental HOMO/LUMO energy levels of these reported HTMs (in Fig. S3†). Hence, the empirical correlations between theoretical $\mathrm{HOMO}($ th.)/LUMO(th.) and experimental HOMO(exp.)/ 
LUMO(exp.) for these HTMs are expressed in the following equations:

$$
\begin{aligned}
& \text { HOMO (exp.) }=0.66 \mathrm{HOMO}(\text { th. })-1.75, R=0.79 \\
& \text { LUMO(exp. })=0.69 \mathrm{LUMO}(\text { th. })-1.07, R=0.88
\end{aligned}
$$

According to eqn (8) and Table $\mathrm{S} 2, \uparrow$ the calculated results show that the values of LUMO energy for Z26 $(-5.08 \mathrm{eV})$ and FDT $(-5.11 \mathrm{eV})$ are in accord with those of the experimental values (Z26: $-5.16 \mathrm{eV}$ (ref. 12) and FDT: $-5.16 \mathrm{eV}$ (ref. 28)), respectively. According to eqn (9) and Table S2, $\uparrow$ the calculated results show that the LUMO values of Z26 $(-2.69 \mathrm{eV})$ and FDT $(-2.28 \mathrm{eV})$ are in accord with those of the experimental values (Z26: $-2.77 \mathrm{eV}$ (ref. 12) and FDT: $-2.28 \mathrm{eV}$ (ref. 28)), respectively. Therefore, eqn (8) and (9) can give an accurate result to predict the HOMO and LUMO energies of the designed HTMs on the basis of the B3P86/6-311G(d,p) calculation. In fact, the HOMO and LUMO energies of HTMs can be evaluated by adiabatic ionization potentials (IPs) and electron affinities (EAs), respectively. ${ }^{25,56}$ The IPs and EAs of the reported Z26 and FDT are calculated by B3P86/6-311G(d,p) functional and basis set. Table $\mathrm{S} 2 \uparrow$ shows that the calculated IPs and EAs of the reported Z26 and FDT are divorced from the HOMO and LUMO energies of these HTMs, respectively. Analogously, in order to further verify the reliability of the theoretical model that was used to predict the HOMO and LUMO energies of the designed HTMs, we calculated the IPs and EAs of the reported arylamine derivativebased HTMs at B3P86/6-311G(d,p) level in comparison with the HOMO and LUMO energies of these HTMs (see Table S1 $\dagger$ ). Hence, two empirical correlation between theoretical IP/EA and experimental HOMO/LUMO of these reported HTMs were fitted (see Fig. S3 and Table S2 $\dagger$ ). As shown in Table S2, $\dagger$ the calculated results reveal that the empirical correlation (Fig. S3c and $\mathrm{d} \dagger$ ) can yield a reliable data about the HOMO and LUMO values of Z26 and FDT, in line with the results from eqn (8) and (9). Therefore, eqn (8) and (9) could be used to predict the HOMO and LUMO energies of the designed HTMs. According to eqn (8) and (9), the calculated values of HOMO and LUMO energies for the designed HTMs (H01-H04) are presented in Fig. 2A.

As shown in Fig. 2A, the values of HOMO energy are H01 $(-5.25 \mathrm{eV}), \mathrm{H} 02(-5.25 \mathrm{eV}), \mathrm{H} 03(-5.26 \mathrm{eV})$ and H04 $(-5.28 \mathrm{eV})$, respectively. For the H01-H04, their HOMO levels are higher than the valence band of the perovskite. Therefore, the HOMO levels of designed HTMs are in good agreement with the valence band of $\mathrm{CH}_{3} \mathrm{NH}_{3} \mathrm{PbI}_{3}(-5.43 \mathrm{eV}$ (ref. 48 and 49)), which ensure a good hole injection from the perovskite to the HTMs. In addition, the illustration of the frontier molecular orbitals in
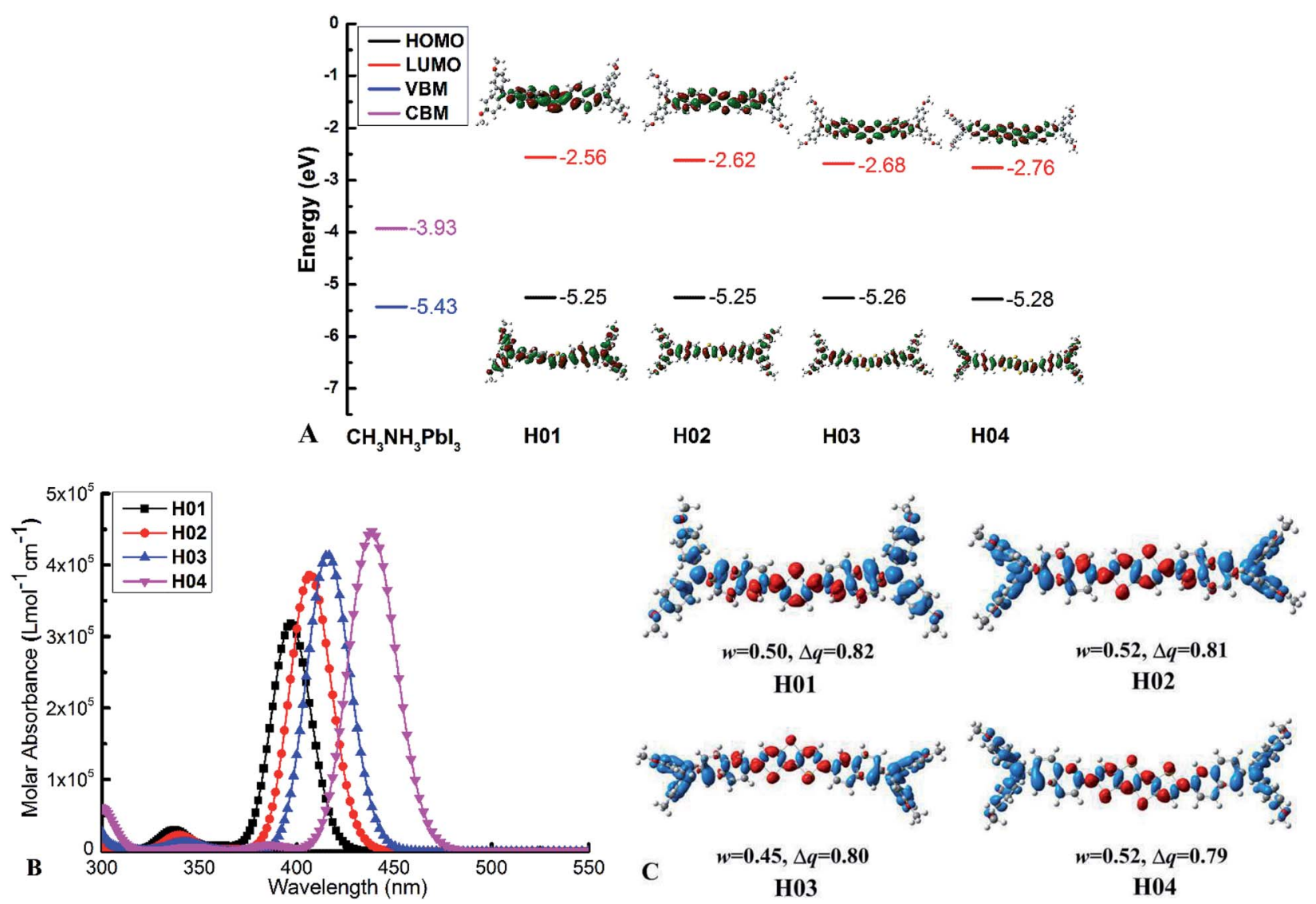

Fig. 2 (A) Energy levels and frontier molecular orbitals of all investigated molecules at the B3P86/6-311G(d,p) levels; (B) simulated absorption spectra of molecules $\mathrm{H01-H04}$ using the TD-BMK/6-31g(d) functional and basis set in tetrahydrofuran; (C) electron density difference plots of electronic transition $\mathrm{S}_{0} \rightarrow \mathrm{S}_{1}$ for $\mathrm{HO1}-\mathrm{HO} 4$ (isovalue: $2 \times 10^{-2} \mathrm{e} \mathrm{au}^{-3}$ ), $w$ is overlap between the regions of density depletion and increment, $\Delta q$ is transferred charge amount $\left(\left|\mathrm{e}^{-}\right|\right)$. 
Fig. 2A indicates that both the HOMOs and LUMOs of H01-H04 are delocalized on the entire molecular chain with $\pi$ characteristics. The HOMO orbital of HTMs with extensive delocalization on the molecular chain is favorable for increasing the electron coupling between the adjacent molecules. ${ }^{18,31}$ According to the HOMO distribution, H01-H04 as HTMs in PSCs may provide large electron coupling that increases the hole mobility.

As shown in Fig. 2A, the LUMO values for $\mathrm{H} 01-\mathrm{H} 04$ are $-2.56,-2.62,-2.68$ and $-2.76 \mathrm{eV}$, respectively. Obviously, the LUMO levels of H01-H04 that are higher than the conduction band of the perovskite (e.g. $\mathrm{CH}_{3} \mathrm{NH}_{3} \mathrm{PbI}_{3}$ ) can effectively prevent the electron from moving from the absorber (e.g., $\mathrm{CH}_{3} \mathrm{NH}_{3}$ $\left.\mathrm{PbBr}_{3}\right)$ to the metal electrodes. Moreover, the energy gaps $\left(E_{\mathrm{g}} \mathrm{s}\right)$ of HTMs are in the following sequence: $2.69 \mathrm{eV}(\mathrm{H} 01)>2.63 \mathrm{eV}$ $(\mathrm{H} 02)>2.58 \mathrm{eV}(\mathrm{H} 03)>2.52 \mathrm{eV}(\mathrm{H} 04)$. The decreased energy gaps from $\mathrm{H} 01$ to $\mathrm{H} 04$ are mainly driven from the downshifted LUMO contributions. Fig. 2A indicates that the LUMO values of the designed H01-H04 are in the order of $\mathrm{H} 01>\mathrm{H} 02>\mathrm{H} 03>$ H04. There is no significant change in the HOMO values of the designed H01-H04. For the designed HTMs, an increased hybridization and $\pi$-conjugation degree of electron-deficiency of $\pi$-bridged units in the LUMO from $\mathrm{H} 01$ to H04 should have a significant impact on the decreasing LUMO level, resulting in the reduction of the band gap. Optimizing electron-deficient $\pi$ bridged units in small-molecule arylamine derivative-based HTMs is an effective strategy to adjust the electronic properties for obtaining suitable frontier molecular orbital levels.

\subsection{Optical absorptions and emission}

Optical properties of HTMs can provide some useful information to evaluate the performance of HTM in PSC devices. Therefore, the optical properties of all HTMs were simulated by the TD-BMK/6-31G(d) functional and basis set on the basis of the ground state structures with the same functional and basis set in tetrahydrofuran solution. Simulated absorption and photoluminescence spectra of Z26 together with the experimental spectra are presented in Fig. $\mathrm{S} 4, \dagger$ which indicates that the TD-BMK/6-31G(d) level in tetrahydrofuran solution can yield an accurate absorption peak in comparison with the experimental one. The optical parameters of H01-H04 in $\mathrm{S}_{0}-\mathrm{S}_{1}$ states, such as absorption wavelength $\left(\lambda_{\text {abs }}\right)$, oscillator strength $(f)$, major configurations, emission wavelength $\left(\lambda_{\mathrm{em}}\right)$ and Stokes shift are presented in Table 1 . In the $S_{0}-S_{1}$ states of the designed HTMs, the absorption is mostly from the HOMO to LUMO transitions. As shown in Table 1 and Fig. 2B, the maximum absorption wavelengths and oscillator strength (in parentheses) of H01-H04 are $397 \mathrm{~nm}$ (2.19), $407 \mathrm{~nm}$ (2.66), $416 \mathrm{~nm}$ (2.86), and $438 \mathrm{~nm}$ (3.08), respectively.

As shown in Table 1 , the $\lambda_{\text {em }}$ values for H01-H04 in the $\mathrm{S}_{0}-\mathrm{S}_{1}$ states are 519, 536, 552 and $561 \mathrm{~nm}$, respectively. Stokes shifts from the maximum absorption wavelength to the emission wavelengths of H01-H04 are 122, 129, 136 and $123 \mathrm{~nm}$, respectively. It has been reported that a large Stokes shifts of organic HTMs can give rise to a large change in the structure between ground and excited states. ${ }^{13}$ This also contributes to the pore filling of the HTM in PSCs by optical post-treatment or
Table 1 The absorption wavelengths $\lambda_{\text {abs }}(\mathrm{nm})$ and emission wavelengths $\lambda_{\text {em }}(\mathrm{nm})$ of molecules $\mathrm{H} 01-\mathrm{HO} 4$ in the $\mathrm{S}_{0}-\mathrm{S}_{1}$ states together with the Stokes shift at TD-BMK/6-31 g(d) levels in tetrahydrofuran solvent

\begin{tabular}{|c|c|c|c|c|c|}
\hline & \multicolumn{3}{|c|}{ Absorption } & \multicolumn{2}{|c|}{ Emission } \\
\hline & $\lambda_{\mathrm{abs}}$ & $f$ & Assignments & $\lambda_{\mathrm{em}}$ & Shift (nm) \\
\hline H01 & 397 & 2.19 & $\mathrm{H} \rightarrow \mathrm{L}(85 \%)$ & 519 & 122 \\
\hline H02 & 407 & 2.66 & $\mathrm{H} \rightarrow \mathrm{L}(84 \%)$ & 536 & 129 \\
\hline H03 & 416 & 2.86 & $\mathrm{H} \rightarrow \mathrm{L}(80 \%)$ & 552 & 136 \\
\hline H04 & 438 & 3.08 & $\mathrm{H} \rightarrow \mathrm{L}(81 \%)$ & 561 & 123 \\
\hline
\end{tabular}

simple annealing. ${ }^{13} \mathrm{H} 01-\mathrm{H} 04$ molecules with large Stokes shifts may improve the performance of HTMs in PSC applications.

To further explore the charge transfer properties of new designed HTMs during the optical excitation process, the overlap between the regions of density depletion and increment $(w)$, transferred charge amount $(\Delta q)$ in $\left(\left|\mathrm{e}^{-}\right|\right)$and electron density difference (EDD) plots of $\mathrm{H} 01-\mathrm{H} 04$ are provided (in Fig. 2C). Fig. 2C shows that the decreased electron densities (blue) are obviously located in the entire chains. The increased electron densities (red) are limited to the electron-deficiency region of $\pi$-bridged units in the molecules. In addition, the corresponding parameters, such as $\Delta q$ and $w$ are presented in Fig. 2C. The $\Delta q$ values of $\mathrm{H} 01-\mathrm{H} 04$ are $0.82,0.81,0.80$ and $0.79\left|\mathrm{e}^{-}\right|$, respectively, and the $w$ values of $\mathrm{H} 01-\mathrm{H} 04$ are 0.50 , $0.52,0.45$ and 0.52 , respectively. The $\Delta q$ and $w$ values of Z26 are 0.78 and $0.50\left|\mathrm{e}^{-}\right|$. For these parameters of EDD, there are no significant differences in comparison with the Z26. This indicates that the designed HTMs can ensure an efficient charge separation, which promotes HTM performance in PSC applications.

\subsection{Solubility and stability of HTMs}

For the fabrication of PSC devices, the HTMs are coated on ITO or perovskite films. In order to ensure that the HTMs can be coated as a uniform film on the ITO or perovskite, a good solubility of the HTMs is needed. Theoretically, solvation free energy $(\Delta G)$ can be used to estimate the solubility of organic materials. ${ }^{57,58} \Delta G$ of organic materials is equal to the difference between the free energy in the solvent and that in the gaseous phase. ${ }^{57,58}$ Herein, tetrahydrofuran was selected as the solvent. Table 2 shows that the calculated $\Delta G$ values of $\mathrm{Z} 26$ and H01$\mathrm{H} 04$ are $-0.54 \mathrm{eV},-0.53 \mathrm{eV},-0.58 \mathrm{eV},-0.66 \mathrm{eV}$ and $-0.65 \mathrm{eV}$,

Table 2 The adiabatic ionization potential ( $I P_{a}$ in eV), electron affinities ( $E A_{a}$ in $\mathrm{eV}$ ), absolute hardness ( $\eta$ in $\mathrm{eV}$ ) and solvation free energy ( $\Delta G$ in $\mathrm{eV}$ ) of the molecules $\mathrm{HO1}-\mathrm{HO} 4$

\begin{tabular}{lrrrr}
\hline & H01 & \multicolumn{1}{c}{ H02 } & \multicolumn{1}{c}{ H03 } & \multicolumn{1}{c}{ H04 } \\
\hline $\mathrm{IP}_{\mathrm{a}}$ & 6.03 & 6.01 & 6.00 & 6.01 \\
$\mathrm{EA}_{\mathrm{a}}$ & 1.51 & 1.58 & 1.67 & 1.80 \\
$\eta$ & 2.26 & 2.21 & 2.17 & 2.10 \\
$\Delta G$ & -12.70 & -13.91 & -15.66 & -15.51
\end{tabular}


respectively. It can be seen that the investigated $\mathrm{H} 01-\mathrm{H} 04$ molecules with similar $\Delta G$ in comparison with that of Z26 (in Table S3†) may exhibit good solubility.

In order to obtain an excellent HTM, stability is a very important parameter. Absolute hardness $(\eta)$ can be used to estimate the stability of an organic material to a certain extent. ${ }^{22}$ $\eta$ can be described by the equation, $\eta=(\mathrm{IP}-\mathrm{EA}) / 2,{ }^{22}$ where IP is the adiabatic ionization potential and EA the adiabatic electron affinity. ${ }^{59}$ As shown in Table 2 , the calculated $\eta$ values of H01$\mathrm{H} 04$ are 2.26, 2.21, 2.17 and $2.10 \mathrm{eV}$, respectively. The values of $\eta$ for the designed HTMs are close to that $(2.06 \mathrm{eV}$ ) of Z26 (in Table S3†). It indicates that these designed HTMs may have good stability.

\subsection{Hole mobility}

Development of HTMs with high hole mobility is an important way to improve the performance of PSC devices. According to the Marcus theory (eqn (5)), the hole reorganization energy $\left(\lambda_{h}\right)$ and hole-transfer integral $\left(v_{\mathrm{h}}\right)$ are key factors affecting the charge-transfer rate. Generally speaking, a small recombination energy is in favor of improving the charge-transfer rate. According to the calculated results presented in Table 3 , the $\lambda_{\mathrm{h}}$ values of HTMs are H01 (0.194 eV), H02 (0.195 eV), H03 (0.188 $\mathrm{eV})$ and H04 $(0.161 \mathrm{eV})$, respectively. In comparison with the $\lambda_{\mathrm{h}}$ value of Z26 $(0.280 \mathrm{eV})$, the designed HTMs with smaller $\lambda_{\mathrm{h}}$ values may improve the charge-transfer rate or hole mobility. This result can be explained by introducing the electrondeficient units in arylamine derivative-based HTMs, which is an effective strategy to reduce the recombination energy.

Another important parameter influencing the holetransporting ability of HTMs is the hole-transfer integral $v_{\mathrm{h}}$. According to Marcus theory (eqn (5)), a maximized $v_{\mathrm{h}}$ value is helpful in promoting the hole-transfer rate of HTM. For HTMs, it is well known that the intermolecular arrangement and frontier molecular orbitals of neighboring molecules are the key factors influencing $v_{\mathrm{h}}{ }^{60}$ The intermolecular arrangement of a HTM can be obtained from the crystal structure. On the basis of the predicted crystal structure of Z26 and designed HTMs, one fragment is arbitrarily chosen as the center. All of the adjacent fragments with the center are defined as the transport pathways; that is, each transport pathway is the paired dimer between neighboring and center molecules. The main pathways of Z26, FDT and H01-H04 are presented in Fig. S5† and 3. Predicted crystal data of investigated molecules are listed in Table S4. $\uparrow$ As shown in Table $\mathrm{S} 4, \uparrow$ the calculated results indicate that the crystal structures of Z26 and H01-H04 with the lowest total energies belong to the space groups of $C_{2}, P_{1}, P_{1}, P n a 2_{1}$ and $P 2{ }_{1} 2_{1} 2_{1}$, respectively. As shown in Fig. S5, $\dagger$ the calculated $u_{\mathrm{h}}$ $\left(8.050 \times 10^{-4} \mathrm{~cm}^{2} \mathrm{~V}^{-1} \mathrm{~s}^{-1}\right)$ of $\mathrm{Z} 26$ has the same order of magnitude as that of the experimental value $\left(1.34 \times 10^{-4} \mathrm{~cm}^{2}\right.$ $\left.\mathrm{V}^{-1} \mathrm{~s}^{-1}\right) .{ }^{12}$ The calculated results indicate that the current

Table 3 The hole reorganization energy $\lambda_{\mathrm{h}}(\mathrm{eV})$, hole coupling $v_{\mathrm{h}}(\mathrm{eV})$, hole transport rate $k_{\mathrm{h}}\left(\mathrm{s}^{-1}\right)$, center-of-mass distance $D(\AA)$ and hole mobility $u_{\mathrm{h}}\left(\mathrm{cm}^{2} V^{-1} \mathrm{~s}^{-1}\right)$ of main hopping pathway selected on basis of the crystal structure for molecules $\mathrm{H} 01-\mathrm{H} 04$

\begin{tabular}{|c|c|c|c|c|c|c|}
\hline Compounds & Pathways & $\lambda_{\mathrm{h}}$ & $v_{\mathrm{h}}$ & $k_{\mathrm{h}}$ & $D$ & $u_{\mathrm{h}}$ \\
\hline \multirow[t]{6}{*}{ H01 } & 1 & \multirow[t]{6}{*}{0.194} & $4.682 \times 10^{-3}$ & $1.28 \times 10^{11}$ & 8.190 & \multirow[t]{6}{*}{$1.372 \times 10^{-2}$} \\
\hline & 2 & & $-5.695 \times 10^{-4}$ & $1.89 \times 10^{9}$ & 15.382 & \\
\hline & 3 & & $1.868 \times 10^{-5}$ & $2.03 \times 10^{6}$ & 15.649 & \\
\hline & 4 & & $7.789 \times 10^{-3}$ & $3.53 \times 10^{11}$ & 8.557 & \\
\hline & 5 & & $-2.000 \times 10^{-5}$ & $2.33 \times 10^{6}$ & 21.941 & \\
\hline & 6 & & $-2.000 \times 10^{-5}$ & $2.33 \times 10^{6}$ & 19.851 & \\
\hline \multirow[t]{9}{*}{ H02 } & 1 & \multirow[t]{9}{*}{0.195} & $3.240 \times 10^{-5}$ & $6.03 \times 10^{12}$ & 4.781 & \multirow[t]{9}{*}{$8.886 \times 10^{-2}$} \\
\hline & 2 & & $1.934 \times 10^{-5}$ & $2.15 \times 10^{6}$ & 21.664 & \\
\hline & 3 & & $1.424 \times 10^{-3}$ & $1.17 \times 10^{10}$ & 20.938 & \\
\hline & 4 & & $-9.939 \times 10^{-4}$ & $5.68 \times 10^{9}$ & 21.288 & \\
\hline & 5 & & $1.560 \times 10^{-4}$ & $1.40 \times 10^{8}$ & 31.839 & \\
\hline & 6 & & $6.650 \times 10^{-4}$ & $2.54 \times 10^{9}$ & 31.826 & \\
\hline & 8 & & $1.794 \times 10^{-5}$ & $1.85 \times 10^{6}$ & 15.843 & \\
\hline & 7 & & $2.000 \times 10^{-5}$ & $2.30 \times 10^{6}$ & 16.993 & \\
\hline & 9 & & $6.151 \times 10^{-4}$ & $2.17 \times 10^{9}$ & 16.093 & \\
\hline \multirow[t]{10}{*}{ H03 } & 1 & \multirow[t]{10}{*}{0.188} & $9.254 \times 10^{-3}$ & $5.36 \times 10^{11}$ & 8.922 & \multirow[t]{10}{*}{$2.624 \times 10^{-2}$} \\
\hline & 2 & & $-3.391 \times 10^{-4}$ & $7.20 \times 10^{8}$ & 17.796 & \\
\hline & 3 & & $1.736 \times 10^{-3}$ & $1.89 \times 10^{10}$ & 12.811 & \\
\hline & 4 & & $6.564 \times 10^{-4}$ & $2.70 \times 10^{9}$ & 13.066 & \\
\hline & 5 & & $-3.833 \times 10^{-5}$ & $9.20 \times 10^{6}$ & 18.186 & \\
\hline & 6 & & $-5.333 \times 10^{-4}$ & $1.78 \times 10^{9}$ & 15.638 & \\
\hline & 7 & & $4.392 \times 10^{-4}$ & $1.21 \times 10^{9}$ & 17.820 & \\
\hline & 8 & & $-5.073 \times 10^{-4}$ & $1.61 \times 10^{9}$ & 18.842 & \\
\hline & 9 & & $-2.419 \times 10^{-4}$ & $3.67 \times 10^{8}$ & 13.993 & \\
\hline & 10 & & $8.037 \times 10^{-4}$ & $4.05 \times 10^{9}$ & 13.993 & \\
\hline \multirow[t]{5}{*}{ H04 } & 1 & \multirow[t]{5}{*}{0.161} & $-3.419 \times 10^{-3}$ & $1.03 \times 10^{11}$ & 9.551 & \multirow[t]{5}{*}{$5.197 \times 10^{-3}$} \\
\hline & 2 & & $-6.399 \times 10^{-5}$ & $3.60 \times 10^{7}$ & 13.104 & \\
\hline & 3 & & $3.000 \times 10^{-5}$ & $7.91 \times 10^{6}$ & 15.375 & \\
\hline & 5 & & $2.179 \times 10^{-4}$ & $4.17 \times 10^{8}$ & 16.407 & \\
\hline & 4 & & $1.717 \times 10^{-3}$ & $2.59 \times 10^{10}$ & 10.373 & \\
\hline
\end{tabular}




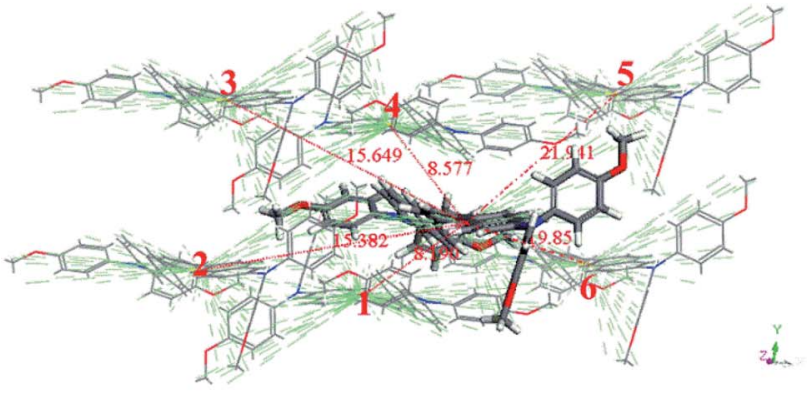

H01

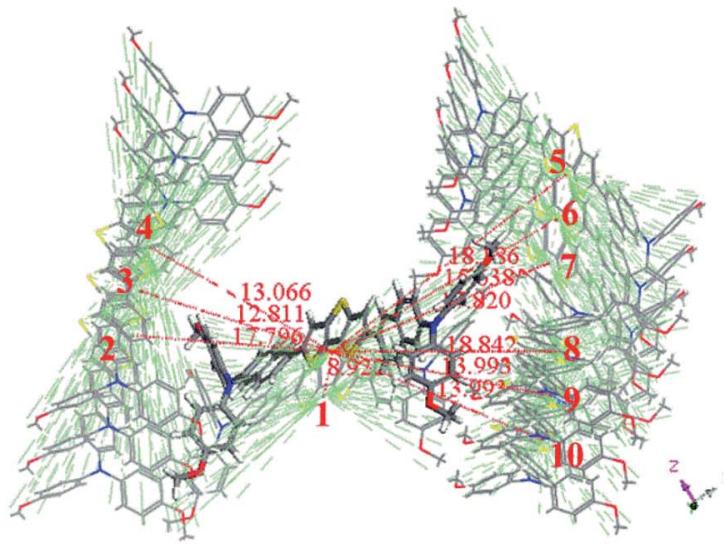

H03

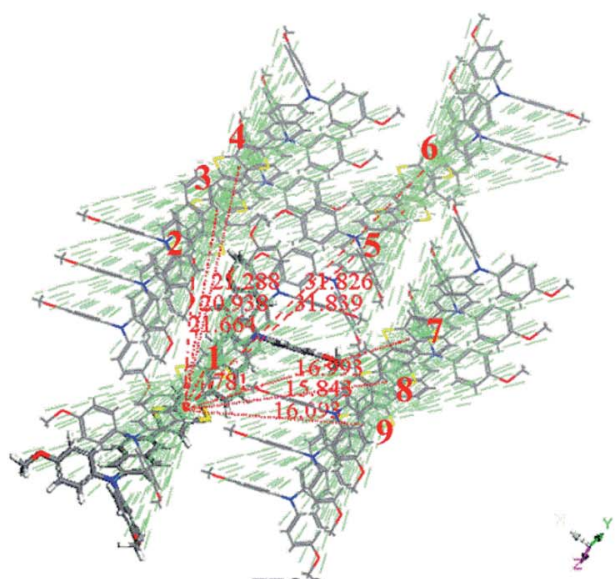

H02

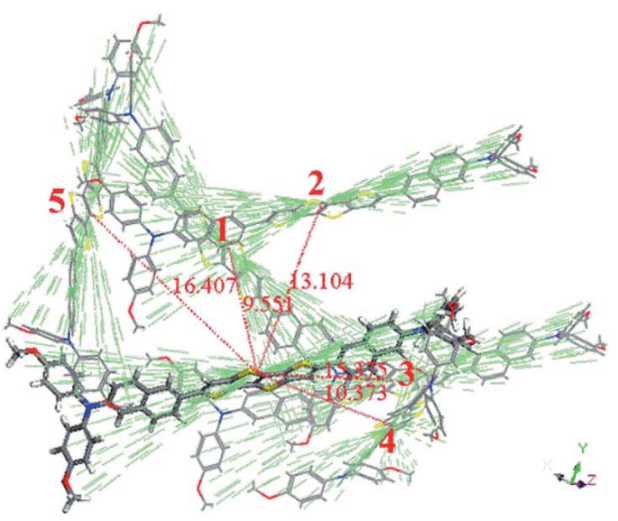

H04

Fig. 3 Main hole hopping pathways selected on basis of the crystal structures for all investigated molecules.

theoretical level in this work is reliable based on the estimation of hole mobility for the designed HTMs.

The reorganization energy $\lambda_{\mathrm{h}}(\mathrm{eV})$, hole coupling $v_{\mathrm{h}}(\mathrm{eV})$, hole transport rate $k_{\mathrm{h}}\left(\mathrm{s}^{-1}\right)$, center-of-mass distance $D(\AA)$ and hole mobility $u_{\mathrm{h}}\left(\mathrm{cm}^{2} \mathrm{~V}^{-1} \mathrm{~s}^{-1}\right)$ of main hopping pathway selected on the basis of the crystal structure for molecules H01-H04 are listed in Table 3. Table 3 shows that the $u_{\mathrm{h}}$ values of H01-H04 are $1.372 \times 10^{-2}, 8.886 \times 10^{-2}, 2.624 \times 10^{-2}$ and $5.197 \times 10^{-3}$ $\mathrm{cm}^{2} \mathrm{~V}^{-1} \mathrm{~s}^{-1}$, respectively. In comparison with the calculated $u_{\mathrm{h}}$ of Z26 $\left(8.050 \times 10^{-4} \mathrm{~cm}^{2} \mathrm{~V}^{-1} \mathrm{~s}^{-1}\right)$ and FDT $\left(2.848 \times 10^{-3} \mathrm{~cm}^{2}\right.$ $\mathrm{V}^{-1} \mathrm{~s}^{-1}$ ) (see Fig. S5 $\dagger$ ), the designed H01-H04 with higher hole mobility may improve the performance of PSC devices.

It can be seen that the $u_{\mathrm{h}}$ values of H01-H04 are higher than those of Z26 and FDT, which can largely promote the performance of HTMs in PSC applications. H01-H04 with high $u_{\mathrm{h}}$ values are attributed to a small recombination energy and large transfer integral. Therefore, in view of high $u_{\mathrm{h}}$ values, the designed H01-H04 as HTMs can act as promising materials for improving the performance of PSCs.

On the basis of investigating the designed $\mathrm{H} 01-\mathrm{H} 04$, it can be concluded that these molecules serve as promising candidates as HTMs for PSC applications. Therefore, our proposed H01-H04 can be synthesized by the judicious design of the route, as shown in Fig. $\mathrm{S} 6, \uparrow$ on the basis of the previously reported procedures. $^{21}$

\section{Conclusions}

In summary, by adjusting the electron-rich arylamine derivatives in thienothiophene derivatives as $\pi$-bridged small molecule HTMs, four HTMs (H01-H04) were built on the basis of the reported excellent Z26. According to the requirement and working mechanism of HTM in the PSC devices, we investigated the electronic properties, optical absorptions, solubility, stability and hole transporting ability of these HTMs using DFT, TD-DFT and Marcus theory. The calculated results indicate that the selected methods we used herein could reproduce reliable HOMO/LUMO energy, absorption maximum and hole mobility of the reported Z26 in line with its experimental values. The frontier molecular orbital levels of molecules H01-H04 match the conduction and valence bands of $\mathrm{CH}_{3} \mathrm{NH}_{3} \mathrm{PbI}_{3}$ very well. Compared with Z26, the designed H01-H04 exhibit good optical properties, better solubility, good stability and higher hole mobilities. The designed HTMs of H01-H04 exhibit high hole mobilities of $1.372 \times 10^{-2}, 8.886 \times 10^{-2}, 2.624 \times 10^{-2}$ and $5.197 \times 10^{-3} \mathrm{~cm}^{2} \mathrm{~V}^{-1} \mathrm{~s}^{-1}$, respectively. Conclusively, the results 
confirmed that the design strategy of adjusting the electron-rich arylamine derivatives in thienothiophene derivatives as $\pi$ bridged HTMs is a reliable approach to obtain the promising HTMs for PSC applications.

\section{Conflicts of interest}

There are no conflicts to declare.

\section{Acknowledgements}

This work was supported by National Natural Science Foundation of China (Grant No. 21803043), Fundamental Research Funds for the Central Universities (Grant No. SWU117050), and Project Funds for Staying (Working) at Chongqing of the Outbound Postdoc.

\section{References}

1 G. Xing, N. Mathews, S. Sun, S. S. Lim, Y. M. Lam, M. Grätzel, S. Mhaisalkar and T. C. Sum, Science, 2013, 342, 344-347.

2 D. Shi, V. Adinolfi, R. Comin, M. Yuan, E. Alarousu, A. Buin, Y. Chen, S. Hoogland, A. Rothenberger and K. Katsiev, Science, 2015, 347, 519-522.

3 Q. Dong, Y. Fang, Y. Shao, P. Mulligan, J. Qiu, L. Cao and J. Huang, Science, 2015, 347, 967-970.

4 N. J. Jeon, H. Na, E. H. Jung, T.-Y. Yang, Y. G. Lee, G. Kim, H.-W. Shin, S. Il Seok, J. Lee and J. Seo, Nat. Energy, 2018, 3, 682-689.

5 X. Sun, D. Zhao and Z. a. Li, Chin. Chem. Lett., 2018, 29, 219231.

6 X. Zhao and M. Wang, Materials Today Energy, 2018, 7, 208220.

7 P.-Y. Chen and S.-H. Yang, Opt. Mater. Express, 2016, 6, 3651. 8 I. García-Benito, I. Zimmermann, J. Urieta-Mora, J. Aragó, J. Calbo, J. Perles, A. Serrano, A. Molina-Ontoria, E. Ortí, N. Martín and M. K. Nazeeruddin, Adv. Funct. Mater., 2018, 28, 1801734.

9 X. Liu, F. Kong, F. Guo, T. Cheng, W. Chen, T. Yu, J. Chen, Z. a. Tan and S. Dai, Dyes Pigm., 2017, 139, 129-135.

10 J. Zhang, T. Zhang, L. Jiang, U. Bach and Y.-B. Cheng, ACS Energy Lett., 2018, 3, 1677-1682.

11 M. Saliba, S. Orlandi, T. Matsui, S. Aghazada, M. Cavazzini, J.-P. Correa-Baena, P. Gao, R. Scopelliti, E. Mosconi, K.-H. Dahmen, F. De Angelis, A. Abate, A. Hagfeldt, G. Pozzi, M. Graetzel and M. K. Nazeeruddin, Nat. Energy, 2016, 1, 15017.

12 F. Zhang, Z. Wang, H. Zhu, N. Pellet, J. Luo, C. Yi, X. Liu, H. Liu, S. Wang, X. Li, Y. Xiao, S. M. Zakeeruddin, D. Bi and M. Grätzel, Nano Energy, 2017, 41, 469-475.

13 H. Li, K. Fu, A. Hagfeldt, M. Grätzel, S. G. Mhaisalkar and A. C. Grimsdale, Angew. Chem., Int. Ed., 2014, 53, 4085-4088. 14 D. Bi, B. Xu, P. Gao, L. Sun, M. Grätzel and A. Hagfeldt, Nano Energy, 2016, 23, 138-144.

15 H. Zhang, Y. Wu, W. Zhang, E. Li, C. Shen, H. Jiang, H. Tian and W. H. Zhu, Chem. Sci., 2018, 9, 5919-5928.
16 P. Agarwala and D. Kabra, J. Mater. Chem. A, 2017, 5, 13481373.

17 R. A. Belisle, P. Jain, R. Prasanna, T. Leijtens and M. D. McGehee, ACS Energy Lett., 2016, 1, 556-560.

18 A. Fakharuddin, L. Schmidt-Mende, G. Garcia-Belmonte, R. Jose and I. Mora-Sero, Adv. Energy Mater., 2017, 7, 1700623.

19 Z.-Z. Sun, Y.-L. Xu, W.-L. Ding and W.-J. Chi, Synth. Met., 2019, 247, 157-162.

20 Y. L. Xu, W. L. Ding and Z. Z. Sun, Nanoscale, 2018, 10, 20329-20338.

21 R. Azmi, S. Y. Nam, S. Sinaga, Z. A. Akbar, C.-L. Lee, S. C. Yoon, I. H. Jung and S.-Y. Jang, Nano Energy, 2018, 44, 191-198.

22 W. J. Chi, Q. S. Li and Z. S. Li, Nanoscale, 2016, 8, 6146-6154.

23 W. J. Chi, P.-P. Sun and Z. S. Li, Nanoscale, 2016, 8, 1775217756.

24 W.-J. Chi, D.-Y. Zheng, X.-F. Chen and Z.-S. Li, J. Mater. Chem. C, 2017, 5, 10055-10060.

25 E. G. Kim, V. Coropceanu, N. E. Gruhn, R. S. SanchezCarrera, R. Snoeberger, A. J. Matzger and J. L. Bredas, J. Am. Chem. Soc., 2007, 129, 13072-13081.

26 W. Hu, Z. Zhang, J. Cui, W. Shen, M. Li and R. He, Nanoscale, 2017, 9, 12916-12924.

27 A. Cohen, P. Mori-Sánchez and W. Yang, Chem. Rev., 2012, 112, 289-320.

28 M. Saliba, S. Orlandi, T. Matsui, S. Aghazada, M. Cavazzini, J.-P. Correa-Baena, P. Gao, R. Scopelliti, E. Mosconi and K.-H. Dahmen, Nat. Energy, 2016, 1, 15017.

29 J. Tomasi, B. Mennucci and R. Cammi, Chem. Rev., 2005, 105, 2999-3094.

30 X. Liu, M. Li, R. He and W. Shen, Phys. Chem. Chem. Phys., 2014, 16, 311-323.

31 M. J. Frisch, G. W. Trucks, H. B. Schlegel, G. E. Scuseria, M. A. Robb, J. R. Cheeseman, G. Scalmani, V. Barone, B. Mennucci, G. A. Petersson, H. Nakatsuji, et al., Gaussian 09, Revision A.01, Gaussian, Inc., Wallingford, CT, 2009.

32 W. Q. Deng, L. Sun, J. D. Huang, S. Chai, S. H. Wen and K. L. Han, Nat. Protoc., 2015, 10, 632-642.

33 J. L. Bredas, D. Beljonne, V. Coropceanu and J. Cornil, Chem. Rev., 2004, 104, 4971-5004.

34 B. C. Lin, C. P. Cheng and Z. P. M. Lao, J. Phys. Chem. A, 2003, 107, 5241-5251.

35 M. Y. Kuo, H. Y. Chen and I. Chao, Chem.-Eur. J., 2007, 13, 4750-4758.

36 L. Wang, G. Nan, X. Yang, Q. Peng, Q. Li and Z. Shuai, Chem. Soc. Rev., 2010, 39, 423-434.

37 J. Bisquert, Phys. Chem. Chem. Phys., 2008, 10, 3175-3194.

38 W. Q. Deng and W. A. Goddard III, J. Phys. Chem. B, 2004, 108, 8614-8621.

39 R. A. Marcus, Annu. Rev. Phys. Chem., 1964, 15, 155-196.

40 R. A. Marcus, Angew. Chem., Int. Ed., 1993, 32, 1111-1121.

41 X. Yang, L. Wang, C. Wang, W. Long and Z. Shuai, Chem. Mater., 2008, 20, 3205-3211.

42 X. Yang, Q. Li and Z. Shuai, Nanotechnology, 2007, 18, 424029. 
43 C. Wang, F. Wang, X. Yang, Q. Li and Z. Shuai, Org. Electron., 2008, 9, 635-640.

44 S.-H. Wen, A. Li, J. Song, W.-Q. Deng, K.-L. Han and W. A. Goddard III, J. Phys. Chem. B, 2009, 113, 8813-8819.

45 K. Senthilkumar, F. Grozema, F. Bickelhaupt and L. Siebbeles, J. Chem. Phys., 2003, 119, 9809-9817.

46 J. P. Perdew, J. Chevary, S. Vosko, K. A. Jackson, M. R. Pederson, D. Singh and C. Fiolhais, Phys. Rev. B, 1992, 46, 6671.

47 G. T. te Velde, F. M. Bickelhaupt, E. J. Baerends, C. Fonseca Guerra, S. J. van Gisbergen, J. G. Snijders and T. Ziegler, J. Comput. Chem., 2001, 22, 931-967.

48 C. F. Guerra, J. Snijders, G. Te Velde and E. Baerends, Theor. Chem. Acc., 1998, 99, 391-403.

49 ADF2014 SCM Theoretical Chemistry, Vrije Universiteit, Amsterdam, The Netherlands, http://www.scm.com.

50 Materials Studio, San Diego, CA, 2005.

51 G. Day, W. Motherwell, H. Ammon, S. Boerrigter, R. Della Valle, E. Venuti, A. Dzyabchenko, J. Dunitz, B. Schweizer and B. Van Eijck, Acta Crystallogr., Sect. B: Struct. Sci., 2005, 61, 511-527.
52 R. Jin and Y. Chang, Phys. Chem. Chem. Phys., 2015, 17, 20942103.

53 W.-J. Chi and Z.-S. Li, Phys. Chem. Chem. Phys., 2015, 17, 5991-5998.

54 S. S. Reddy, K. Gunasekar, J. H. Heo, S. H. Im, C. S. Kim, D. H. Kim, J. H. Moon, J. Y. Lee, M. Song and S. H. Jin, Adv. Mater., 2016, 28, 686-693.

55 S. Tze Chien, C. Shi, X. Guichuan, L. Xinfeng and W. Bo, Nanotechnology, 2015, 26, 342001.

56 D. A. Egger, S. Weissman, S. Refaely-Abramson, S. Sharifzadeh, M. Dauth, R. Baer, S. Kummel, J. B. Neaton, E. Zojer and L. Kronik, J. Chem. Theory Comput., 2014, 10, 1934-1952.

57 J. M. Ho, A. Klamt and M. L. Coote, J. Phys. Chem. A, 2010, 114, 13442-13444.

58 Y. Zhang, Y. Y. Li, C. Chen, L. Wang and J. L. Zhang, Org. Electron., 2017, 49, 255-261.

59 H. Liu and X. Liu, J. Mater. Chem. C, 2018, 6, 6816-6822.

60 A. Zhugayevych, O. Postupna, R. C. Bakus II, G. C. Welch, G. C. Bazan and S. Tretiak, J. Phys. Chem. C, 2013, 117, 4920-4930. 